\title{
Revisiones
}

\section{El cuadro clínico de la intoxicación ocupacional por plomo}

\author{
Augusto V. Ramírez ${ }^{1}$
}

"Si juzgásemos el interés que algún asunto médico despierta por el número de escritos que ha merecido, no tendríamos más que considerar a la intoxicación por plomo como el más importante de todos aquellos que han sido tratados hasta hoy"

Orfila, 1817

Resumen Al plomo, metal pesado grisáceo y ubicuo, se le ha encontrado en lugares tan inverosímiles como los hielos fósiles de Groenlandia. Egipcios y hebreos lo usaron. Los fenicios c. 2000 AC trabajaron menas de plomo en España. Al final del s XX, en países desarrollados, la intoxicación por plomo pasó de problema ocupacional a problema de salud pública. Entre nosotros aún es frecuente la intoxicación ocupacional. El diagnóstico pasa por reconocer la existencia de plomo en el ambiente laboral y en tomar una buena historia clínica y ocupacional. El diagnóstico diferencial considera cuadros dolorosos neurológicos y abdominales. Plomo-sanguíneo y zinc-protoporfirina son análisis cruciales y fidedignos. En expuestos, el plomo-sanguíneo suele llegar a $40 \mathrm{ug} / 100 \mathrm{~mL}$, aunque en trabajadores de industrias insalubres puede alcanzar $80 \mathrm{ug} / 100 \mathrm{~mL}$ y la zinc-protoporfirina ser mayor de $4 \mathrm{ug} / \mathrm{g}$ de hemoglobina. El tratamiento se basa en quelar el plomo casi específicamente con edetato disódico monocálcico, sin olvidar la penicilamida ni los nuevos ácido dimercapto-succínico y ácido dimercapto-propanosulfónico, que han mejorado las opciones terapéuticas, son menos tóxicos y más fáciles de administrar. Se resalta la importancia del problema plomo como prioritario en salud pública y ocupacional, además de coadyuvar al grave problema ecológico actual.

Palabras clave Plomo; envenenamiento por plomo; exposición ocupacional; exposición a riesgos ambientales.

\section{Occupational lead poisoning}

\section{Abstract}

Lead, a ubiquitous heavy metal, has been found in places as unlikely as Greenland's fossil ice. Egyptians and Hebrews used it. In Spain, Phoenicians c. 2000 BC worked ores of lead. At the end of the XX century, occupational lead's poisoning became a public health problem in developed countries. In non-developed countries occupational lead poisoning is still frequent. Diagnosis is directed to recognize lead existence at the labor environment and good clinical and occupational documentation. Differential diagnosis

1 Médico del Trabajo. American College of Occupational and Environmental Medicine. considers neurological and abdominal pain syndromes. Both blood lead and zinc-protoporphyrin levels are trustworthy and crucial analysis. In exposed workers, blood-lead can attain $40 \mathrm{ug} / 100 \mathrm{~mL}$ and even $80 \mathrm{ug} / 100$ $m L$ in unhealthy industries workers and zincprotoporphyrin is above $4 \mathrm{ug} / \mathrm{g}$ of hemoglobin. Treatment consists mainly in calcium disodium edetate, d-penicillamid lead's quelantes, or the new di-mercapto succinic acid and 2-3 di-mercapto-1-propane sulfonic acid that have improved the therapeutic options, are less toxic and easier to manage. We highlight lead's problem as a high-priority problem in public and occupational health, as well as a serious ecological problem.

Keywords: Lead; lead poisoning; occupational exposure; enviromental exposure . 


\section{INTRODUCCIÓN}

El plomo es un metal grisáceo, maleable y uno de los primeros en ser usados por el hombre. Evidencia hay que ya era conocido en Asia Menor oriental allá por el año 4000 AC. Egipcios y hebreos usaron el plomo y los fenicios trabajaron menas de plomo en España c. 2000 AC. Hipócrates de Cos (370 AC) fue el primero en describir síntomas en trabajadores con plomo. Nicanor, en el s. II AC, relacionó directamente estreñimiento, cólico, palidez, parálisis y perturbaciones de la visión con la exposición al plomo. Plinio el viejo y Paracelso describen el envenenamiento por plomo en los constructores de naves. Dieciséis siglos después, Bernardo Ramazzini, en 1713, describe temblor y parálisis en las manos en alfareros que usan plomo para el glaseado. El primer autor moderno que describió este envenenamiento fue Tanquerel des Planches en un estudio publicado en 1839, basado en 1200 casos de intoxicación por plomo, tan completo que hasta hoy es muy poco lo que se ha agregado a la descripción de síntomas y signos por esta intoxicación. Laennec, en 1831, Andral y Gavarret, en 1840, llaman la atención sobre la anemia causada por el plomo. Garrod, en 1892, fue el primero en informar del aumento porfirinas en la orina; y Behrend, en 1899, observó el punteado basófilo en los hematíes $\left({ }^{1,2}\right)$.

En los 1960, la intoxicación por plomo en los países desarrollados pasó de problema ocupacional a problema de salud pública, sobre todo en infantes, pues el niño puede intoxicarse por ingestión de residuos de pinturas que contengan plomo, por vivir en ciudades industriales contaminadas o en zonas aledañas a depósitos de relaves de plomo. Por este motivo, pediatras o neurólogos eventualmente pueden encontrar este cuadro en su práctica. Sin embargo, quienes sí deben tener una visión firme y exacta de este cuadro clínico son los médicos de empresas que procesan o manejan plomo. Por último, es necesario relevar la importancia del plomo como problema prioritario en salud pública, además de su implicancia ecológica $\left(^{3-5}\right)$.

\section{EXPOSICIÓN AL PLOMO}

El plomo es un metal pesado ubicuo. Se le ha encontrado en lugares tan inverosímiles como en los hielos fósiles de Groenlandia. Actualmente, se halla tanto en el ambiente urbano como rural. Sin embargo, las principales exposiciones proceden del ambiente laboral, sin olvidar la importancia toxicológica de las exposiciones domésticas y alimentarias $\left({ }^{6-8}\right)$.

\section{EXPOSICIÓN OCUPACIONAL}

Los problemas de salud laboral causados por plomo suceden principalmente en la metalurgia primaria y secundaria y en minería extractiva, así como en la industria informal de fabricación de acumuladores eléctricos por extracción secundaria de plomo a partir de baterías recicladas $\left({ }^{8}\right)$.

\section{EXPOSICIÓN DOMÉSTICA}

El principal problema extralaboral es la exposición doméstica en niños con pica que ingieren tierra o pinturas contaminadas con plomo inorgánico, pero también en niños y adultos que ingieren alimentos contaminados, por ejemplo, pan "coloreado" con compuestos de plomo. En los años 60's, en el Hospital 2 de Mayo fuimos testigos de varias intoxicaciones con plomo por comer "pan de yema" de fabricación artesanal coloreado con "amarillo de plomo y cromo", para darle el aspecto amarillo del huevo.

Fuentes domésticas de ingesta de plomo las encontramos en alimentos cocinados o guardados en utensilios de alfarería glaseada o cristal emplomado y en las bebidas alcohólicas de fabricación clandestina destiladas en serpentines de plomo o depositadas en recipientes de ese metal. Este problema data por lo menos de los tiempos romanos y fue precisamente una de las posibles causas de la caída de ese imperio. Intoxicaciones contemporáneas en países desarrollados pueden ser atribuidas a distribución de alimentos en envases o envoltorios que contienen plomo. No olvidemos las drogas ilícitas contaminadas y plomo liberado de balas o esquirlas alojadas en el cuerpo. En algunos países del tercer mundo, se usa maquillaje que contiene plomo en los cosméticos, por ej. "Azarcon" y "Greda” en México, "Pay-loo-ah" del Sudeste asiático y "Maha Yogran Guggulu" de la India $\left({ }^{9-16}\right)$. Tabla 1.

\section{EL AGUA COMO FUENTE DE EXPOSICIÓN}

Otra fuente doméstica importante de ingesta de plomo, es el agua "potable", a partir de uniones de las 
Tabla 1. Fuentes de exposición a plomo.

\begin{tabular}{|c|c|c|c|}
\hline Ocupacional & Ambiental & Abuso de sustancias & Otras \\
\hline $\begin{array}{l}\text { * Fontanería } \\
\text { * Plomería } \\
\text { * Metalurgia de plomo } \\
\text { * Minería de plomo } \\
\text { * Soldadores } \\
\text { * Construcción civil } \\
\text { * Industria cerámica } \\
\text { * Manufactura caucho } \\
\text { * Manufactura de vidrio } \\
\text { * Reparación de buques } \\
\text { * Cortadores de metal } \\
\text { * Manufactura de plásticos } \\
\text { * Manufactura de baterías }\end{array}$ & $\begin{array}{l}\text { * Casas pintadas con } \\
\text { pinturas de plomo } \\
\text { * Industria gasolinas } \\
\text { con plomo } \\
\text { * “Agua potable” } \\
\text { contaminada } \\
\text { * Polvos de suelos cercanos a: } \\
\text { - Fundiciones, puertos } \\
\text { o autovías } \\
\text { - Grifos de venta de } \\
\text { gasolinas con plomo }\end{array}$ & $\begin{array}{l}\text { * Sustancias ilegales: } \\
\text { marihuana, cocaína, } \\
\text { meta-anfetamina } \\
\text { * Licores caseros } \\
\text { * “Oledores” de } \\
\text { gasolina }\end{array}$ & $\begin{array}{l}\text { * Suplementos } \\
\text { vitamínicos } \\
\text { * Soldadura } \\
\text { casera (electrónica) } \\
\text { * Cerámica } \\
\text { glaseada } \\
\text { * Proyectiles } \\
\text { alojados en } \\
\text { el cuerpo } \\
\text { * Pesca: } \\
\text { pesos de plomo } \\
\text { * Reparadores de } \\
\text { carros y botes }\end{array}$ \\
\hline
\end{tabular}

cañerías o de tanques de almacenamiento. El nivel aceptable de plomo $(\mathrm{Pb})$ en agua potable (EE UU, 1991) es 15 ug de $\mathrm{Pb} / \mathrm{L}$; naturalmente, la tendencia es eliminar completamente el plomo del agua de bebida $\left({ }^{17}\right)$.

\section{METABOLISMO}

El metabolismo del plomo es muy complejo y merece un capítulo exclusivo; sin embargo, hacemos aquí un apretado resumen.

\section{ABSORCIÓN, CIRCULACIÓN, DISTRIBUCIÓN Y ALMACENAMIENTO}

El organismo absorbe el plomo inorgánico por las vías respiratoria y gastrointestinal. El plomo orgánico también se absorbe por piel. La vía más importante desde el punto de vista ocupacional es la respiratoria. El plomo tiene gran afinidad por los eritrocitos: el $95 \%$ de la fracción circulante de plomo se une a ellos. El mecanismo por el cual el plomo se liga al hematíe, cómo se libera y cómo se transfiere a los tejidos no está bien dilucidado. Al parecer, el primer lugar de fijación es la membrana celular. No se conoce a qué está unida la pequeña fracción de plomo que permanece en el plasma, aunque es posible que tenga mayor importancia toxicológica que la parte que ingresa a la célula, debido a que el plomo dentro del hematíe tiene menor poder de difusión y por tanto de causar daño. Usualmente encontramos que los niveles de plomo plasmático son moderados, quizá por lo difícil que es analizarlos, lo que torna incierto relacionarlos con las manifestaciones tóxicas $\left({ }^{18}\right)$.

La sangre transporta el plomo hacia todo el cuerpo y lo deposita en los tejidos de acuerdo a una gradiente de concentración y a la afinidad específica de cada órgano. La cantidad total de plomo presente en el organismo en un determinado momento se llama "carga corporal de plomo" (CCP).

Es destacable la afinidad del plomo por el tejido óseo. Se calcula que aproximadamente el $90 \%$ de la CCP se une firmemente a este tejido. Los huesos largos, en sus epífisis, contienen mayor cantidad que los planos, mas el tejido dental es el que contiene más plomo. La concentración máxima de plomo en huesos se alcanza hacia la quinta o sexta década, para luego disminuir. En el organismo, al plomo se le encuentra en equilibrio dinámico con una cinética compleja. La concentración de plomo en sangre o tejidos en un momento dado resulta de una función multivariable determinada por su absorción, excreción y otros parámetros fisiológicos, como su traslado dinámico de y hacia los tejidos $\left({ }^{18-20}\right)$.

Efectos toxicológicos importantes de la acción del plomo en el organismo humano son: 1) Atravesar la barrera placentaria: el plomo comienza a ser detectado en el feto entre las 12 y 14 semanas de gestación, incrementándose conforme avanza su maduración. La distribución corporal en el feto es similar al adulto. 2) En madres expuestas, aumento de abortos espontáneos 
y aumento de morbimortalidad perinatal. 3) En exposición paterna, hipospermia e incremento de abortos en su pareja. 4) Atravesar la barrera hematoencefálica. 5) Experimentalmente producir cáncer en animales (categoría A3 ACGIH). Estudios epidemiológicos en humanos han encontrado aumento significativo de varios tipos de cáncer (estómago, pulmón y vejiga), pero aún no hay nada definitivo respecto a eventuales acciones cancerígenas o mutagénicas del plomo en el hombre $\left({ }^{19,20}\right)$.

\section{EXCRECIÓN}

El plomo se excreta por diferentes vías, pero sólo la renal y la gastrointestinal son de importancia toxicológica. Las heces siempre contienen plomo que proviene en su mayor parte de la fracción no absorbida y de otras fuentes, como: 1) Secreción activa o pérdida pasiva de plomo desde glándulas salivales, páncreas y pared intestinal; 2) Pérdida por reboce desde células epiteliales; y, 3) Excreción biliar, cuyo papel en fisiología humana aún es incierto $\left({ }^{20}\right)$.

Sin embargo, la excreción renal es la de mayor importancia toxicológica debido a que se hace por filtración glomerular. El papel de la reabsorción tubular no está totalmente elucidado. Otras posibles vías de excreción incluyen sudor, pelo, uñas, células epiteliales descamadas, dientes y leche; de esta última se sabe que hay relación directa entre concentración de plomo en sangre y leche de madres expuestas ocupacional o ambientalmente $\left({ }^{20}\right)$.

No se conoce exactamente la vida biológica media del plomo, pero se acepta variaciones amplias entre 15 y 27 años en adultos. Actualmente, se sabe que el plomo está presente en el cuerpo en por lo menos cuatro compartimentos: 1) Compartimiento de intercambio rápido: sangre; 2) Compartimiento de recambio medio: piel, órganos internos blandos y músculos; 3) Compartimiento de recambio lento, representado por la fracción intercambiable de plomo de huesos planos y diáfisis de huesos largos; y, 4) Compartimiento de recambio muy lento: extremos de huesos largos. Como vemos, el plomo requiere de un modelo metabólico muy complejo que explique la cinética de la excreción $\left({ }^{21}\right)$.

La Figura 1 muestra un modelo sencillo del metabolismo del plomo en el ser humano, tomado y adaptado de Hemberg.

\section{ENFERMEDAD OCUPACIONAL POR EXPOSICIÓN A PLOMO}

A la enfermedad producida por exposición laboral a plomo se le llama saturnismo (CIE 10: T 56,0), que se le define como el cuadro clínico caracterizado por síntomas y signos propios más indicadores biológicos de exposición (IBE) compatibles. El diagnóstico no cabe en: 1) Ausencia de enfermedad manifiesta; y, 2) Ausencia de exposición al plomo. La definición elimina casos que son informados como tales, en ausencia de exposición o tan sólo con síntomas aislados. Se acepta sí que, antes de que los síntomas sean manifiestos, existe ya un cuadro subclínico con deterioro a nivel enzimático $\left({ }^{18-20}\right)$.

\section{CUADRO CLÍNICO}

Como premisa, es necesario establecer que en exposición laboral a plomo no existe intoxicación aguda sino intoxicación crónica, y a ésta la podemos dividir en estadios o fases. A la primera fase de la enfermedad se la llama intoxicación "leve" o "subclínica" por plomo y no sólo "absorción aumentada de plomo", como eufemísticamente a veces se le nombra, pues esta acepción no nos dice nada sobre el daño bioquímico ya producido. Reservamos la denominación saturnismo o intoxicación clínica por plomo para las fases más severas. Tampoco debemos aceptar a la intoxicación subclínica como si no pasara nada y cualquiera que sea el vocablo empleado para denominar esta fase, salud ocupacional e higiene industrial deberían ya aplicar normas correctivas en la fuente, el medio o el trabajador, y en ese orden, no a la inversa $\left({ }^{21}\right)$.

Los primeros efectos de exposición a plomo pueden ocurrir incluso en trabajadores completamente asintomáticos, en quienes se puede evidenciar alteraciones en los indicadores biológicos de exposición (IBE), como inhibición de la actividad de la dehidratasa del ácido amino levulínico (AAL-D) y un poco después aumento de excreción urinaria de ácido amino levulínico (AAL) y coproporfirina urinaria (CPU). Estas alteraciones se pueden dar con niveles de plomo sanguíneo $(\mathrm{Pb}-\mathrm{S})$ de $40 \mathrm{ug} / 100 \mathrm{~mL}$. A menudo, los síntomas comienzan en forma insidiosa y pueden pasar desapercibidos hasta que por algún motivo se interrumpe la exposición por un tiempo breve, vacaciones por ej.; entonces, el trabajador se da cuenta de los cambios. El cuadro se inicia con disminución 


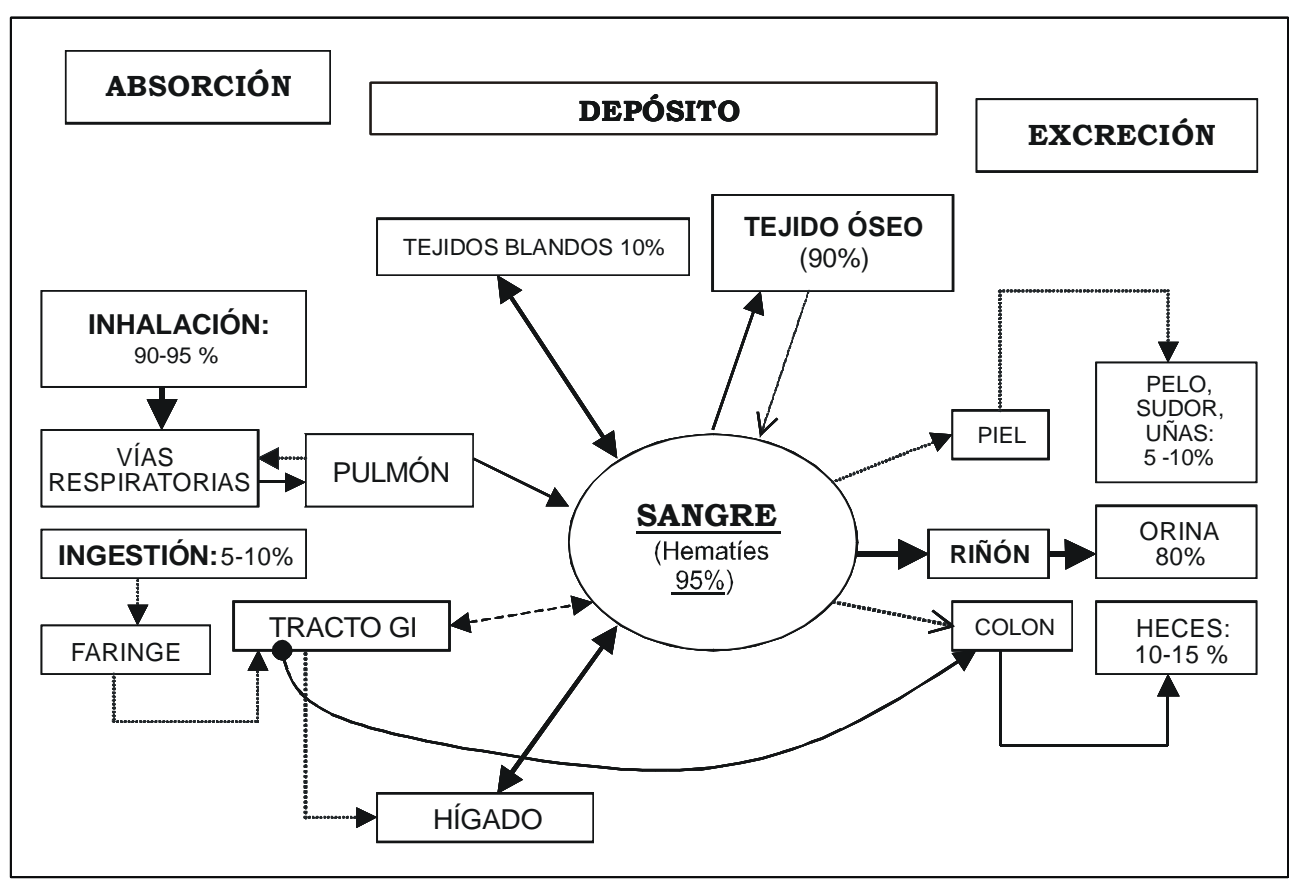

Figura 1. Modelo metabólico del plomo en el ser humano.

subjetiva del bienestar, síntomas vagos como cansancio al final del día, fatiga fácil, insomnio y en general síntomas leves pero acompañados de evidente, definida y constante alteración bioquímica. Los síntomas más tempranos suelen ser difusos. Progresivamente, el trabajador se muestra malhumorado, irritable y se fatiga fácilmente. A menudo pierde interés en las actividades propias de su tiempo de descanso $\left({ }^{21,22}\right)$.

Al continuar la absorción o si la exposición aumenta bruscamente, aparecen síntomas más definidos, como insomnio severo, cefalea, sabor dulce y metálico, algunas veces relacionado con fumar. En esta fase, predominan los síntomas gastrointestinales, los que incluyen pérdida de apetito, malestar epigástrico, meteorismo, estreñimiento o diarrea, dolor muscular difuso, hipersensibilidad en articulaciones y entumecimiento de miembros inferiores. Al examen clínico se puede encontrar temblor fino e hiperreflexia con neuropatía periférica leve detectable con electromiografía. El $\mathrm{Pb}-\mathrm{S}$ puede alcanzar los $80 \mathrm{ug} /$ $100 \mathrm{~mL}$ y el plomo en el ambiente laboral (Pb-A) suele hallarse entre 0,3 y $0,5 \mathrm{mg} / \mathrm{m}^{3}$. Algo común en esta fase, sobre todo a nivel del mar, es la anemia con actividad eritrocitaria del AAL inhibida casi totalmente.
Completa el cuadro AAL y CPU elevadas: 40 ó 50 $\mathrm{mg} / \mathrm{L}$ y 1000 ó $2000 \mathrm{ug} / \mathrm{L}$, respectivamente $\left({ }^{23}\right)$.

Si no se toma acción correctiva alguna, el siguiente paso en clínica es el cólico saturnino. En esta fase, los parámetros hematológicos están francamente alterados. La hemoglobina, a nivel del mar, cae a cerca de $8 \mathrm{~g} / 100$, pero en la altura raramente baja a menos de 13. Sin embargo, el AAL urinario suele ser mayor de $100 \mathrm{mg} / \mathrm{L}$ y la CPU está entre 5000 y $7000 \mathrm{ug} / \mathrm{L}$, mientras la zinc proto-porfirina o ferroporfirina eritrocitaria (ZPP / FEP) excede los 500 ug/100 mL. En esta fase, el plomo puede alcanzar los $100 \mathrm{ug} / 100 \mathrm{~mL}$, sobre todo si hay cólico persistente o recurrente. Aquí, el riesgo de paresia de nervios periféricos, encefalopatía y nefropatía es mayor. De acuerdo a nuestra experiencia, las manifestaciones serias de la intoxicación con plomo son poco frecuentes o raras hoy en día, pero las podemos encontrar sobre todo si el trabajador expuesto es además alcohólico. Cuando accidentalmente se ingiere compuestos de plomo o en exposiciones masivas por vía respiratoria, el cuadro es muy agudo y la encefalopatía se puede desarrollar en semanas e incluso días, predominando el cuadro 
hemático con anemia hemolítica y, en casos extremos, hemólisis y elevación de bilirrubinas séricas $\left({ }^{21,24-27}\right)$.

Un acápite aparte, en niños la sintomatología inicial normalmente incluye vómitos, anorexia, apatía, irritabilidad, fallas en la coordinación, pérdida de las habilidades adquiridas más recientemente, desórdenes de conducta, como agresividad; en general, el cuadro es dramático y severo $\left.{ }^{28}\right)$.

Con la finalidad de resumir, al cuadro de intoxicación ocupacional por plomo lo podemos dividir arbitrariamente en las 3 fases:

\section{FASE DE INTOXICACIÓN SUBCLÍNICA}

Ningún efecto o efectos mínimos sobre la salud del trabajador. Puede incluir inhibición parcial de la AAL$D$ eritrocitaria y reticulocitosis ligera, aumento discreto en la excreción de AAL y más leve aún de ZPP. El $\mathrm{Pb}-\mathrm{S}$ se halla alrededor de los $40 \mathrm{ug} / 100 \mathrm{~mL}$; no olvidemos que en este escrito hablamos sólo de exposición ocupacional. Esta fase todavía podría ser considerada "aceptable". Relativamente nuevas son las investigaciones que hablan de alteraciones neurocomportacionales tempranas detectables con pruebas psicológicas.

\section{INTOXICACIÓN MODERADA}

Predominan síntomas leves, como cansancio, disminución de apetito o malestar epigástrico, en combinación con anemia ligera, excreción elevada de AAL y CPU; la disminución de la velocidad de conducción en nervios periféricos es clara. El Pb-S puede oscilar entre 40 y $60 \mathrm{ug} / 100 \mathrm{~mL}$.

\section{INTOXICACIÓN CLÍNICA}

Encontramos el cuadro clínico clásico con todo el cortejo de signos y síntomas que caracterizan a la intoxicación. El Pb-S siempre es mayor de 70 ug/100 mL. Los otros IBE también están elevados. El tratamiento en esta fase es obligatorio.

Es oportuno recordar que no existe "frontera mágica" alguna que separe estas divisiones un tanto empíricas y que las anormalidades bioquímicas sin exposición no pueden ser consideradas signos de intoxicación por plomo. El problema radica en definir cuándo la desviación mínima de un valor "normal" es inaceptable, por lo que el diagnóstico en las fases iniciales es más una cuestión de criterio del médico ocupacional que de una tabla de valores de laboratorio, por más respaldo científico que tenga $\left({ }^{29-31}\right)$.

La Tabla 2 muestra el resumen de los BEIs que sirve de guía para clasificar esta compleja situación.

\section{DIAGNÓSTICO}

Sin temor a equivocarnos, el diagnóstico certero de intoxicación ocupacional por plomo es difícil y, a pesar de lo sofisticado del apoyo de laboratorio actual, aún tenemos muchos casos de diagnósticos errados o de subdiagnósticos. El error más común es la incapacidad para reconocer la intoxicación cuando está presente, pero lo inverso también es válido. Un problema frecuente del médico ocupacional es confundir otras enfermedades con intoxicación por plomo; el caso de la apendicitis es frecuente. La premisa para el diagnóstico de intoxicación es estar consciente de la existencia de plomo en el ambiente de labor, continuar con algo básico en medicina: una buena historia clínica y ocupacional con un detalle tal, que nos permita valorar el impacto que el xenobiótico podría estar causando. Como sabemos, existen ocupaciones de mayor riesgo y por tanto con mayor probabilidad de causar intoxicación. Por ello, al evaluar al trabajador, no omitir alguna fuente de exposición, sin olvidar las extralaborales. Entonces, sospechar que el plomo es el causante del cuadro clínico actual es crucial, porque podremos dirigir las pruebas de laboratorio específicas en esa dirección y de éstas el $\mathrm{Pb}-\mathrm{S}$ es la más importante, pues no puede haber intoxicación actual si el plomo no está elevado y, también, aunque nos parezca de perogrullo, a mayor nivel de $\mathrm{Pb}-\mathrm{S}$, mayor probabilidad de intoxicación $\left({ }^{1,18}\right)$.

Todas las fases de intoxicación antes descritas presuponen por definición deterioro funcional. Además del $\mathrm{Pb}-\mathrm{S}$, la documentación de estas alteraciones es obligatoria: hemoglobina, reticulocitos, punteado basófilo en hematíes (que todavía se usa a pesar de no ser específica) y AAL-D; en orina AAL-U y CPU. Otro análisis importante a documentar es la ZPP/ FEP, que siempre está elevada en la intoxicación por plomo $\left({ }^{32}\right)$.

La combinación de actividad deprimida de la DAAL y aumento de excreción de AAL y de ZPP/FEP es casi específico en la intoxicación por plomo. Como lineamiento general, podemos decir que todo trabajador expuesto a plomo tiene nivel de ZPP igual o mayor a 
Tabla 2. Valores de Pb-S y otros BEIs aceptados en salud ocupacional.

\begin{tabular}{|c|c|c|}
\hline \multirow[t]{2}{*}{ Indicador } & \multicolumn{2}{|c|}{ Valor considerado normal } \\
\hline & $\begin{array}{l}\text { Gestantes y mujeres } \\
\text { en edad fértil }\end{array}$ & $\begin{array}{c}\text { Expuestos } \\
\text { ocupacionalmente }\end{array}$ \\
\hline $\mathrm{Pb}$ en sangre & $<20 \mathrm{ug} / \mathrm{dL}$ & $\geq 40 \mathrm{ug} / \mathrm{dL}$ \\
\hline Pb urinario (sólo en exposición a Pb alquilo) & $<70 \mathrm{ug} / \mathrm{L}$ & $\geq 150 \mathrm{ug} / \mathrm{L}$ \\
\hline $\begin{array}{l}\text { Zinc proto porfirina o protoporfirina } \\
\text { eritrocitaria libre ZPP/FEP }\end{array}$ & < 75 ug/100 mL hematíes & $\begin{array}{c}\geq 300 \mathrm{ug} / 100 \mathrm{~mL} \mathrm{o} \\
\geq 4 \mathrm{ug} / \mathrm{g} \mathrm{Hb}\end{array}$ \\
\hline Creatinina en orina & \multicolumn{2}{|c|}{$0,4-1,8 \mathrm{~g} / 24 \mathrm{~h}$} \\
\hline Proteínas totales en orina & \multicolumn{2}{|c|}{ Hasta $140 \mathrm{mg} / 24 \mathrm{~h}$} \\
\hline Copro porfirinas urinarias (CPU) & $<100 \mathrm{ug} / \mathrm{L}$ & $\geq 500 \mathrm{ug} / \mathrm{L}$ \\
\hline$\beta-2$ microglobulina $(\beta-2 \mathrm{M})$ & $<250 \mathrm{ng} / \mathrm{mL}$ & $\geq 400 \mathrm{ng} / \mathrm{mL}$ \\
\hline Ácido amino levulínico en orina (ALA-U) & $<4,5 \mathrm{mg} / \mathrm{g}$ de creatinina & $\geq 10 \mathrm{mg} / \mathrm{g}$ de creatinina \\
\hline
\end{tabular}

$80 \mathrm{ug} / 100 \mathrm{~mL}$. Inclusive, si la exposición cesa, la FEP continúa elevada proporcionalmente al nivel de $\mathrm{Pb}-\mathrm{S}$ actual. Existen otros exámenes de laboratorio de menor especificidad, pero que pueden coadyuvar al diagnóstico, como tiempo de vida del hematíe, velocidad de conducción en nervios periféricos, sobre todo en los del grupo muscular que más usa el trabajador, pruebas neuro-comportacionales, pruebas de función renal, etc. $\left.{ }^{33-36}\right)$.

En cambio, la histórica línea de Burton no es indicadora de intoxicación, sino mas bien de exposición y de mala higiene bucal. Como siempre sucede en medicina, aquí tampoco existe prueba alguna con un valor límite que nos asegure un borderline entre intoxicación y no intoxicación por plomo, de manera que lo que nos lleve a establecer el diagnóstico será siempre la valoración sopesada de datos diferentes pero complementarios, tal la historia positiva de exposición, los signos y síntomas descritos, a los que añadiremos los resultados de laboratorio, complementados por lo menos con una onza de juicio clínico $\left({ }^{37}\right)$.

\section{DIAGNÓSTICO DIFERENCIAL}

El primer punto a considerar en el diagnóstico diferencial es el de los falsos diagnósticos de intoxicación por plomo. Hasta hace poco era común ver en los hospitales mineros cómo el clásico cólico saturnino, un cólico "seco" sin diarrea, era confundido en patología abdominal, con cuadros que incluían apendicitis aguda, úlcera gástrica o duodenal, porfiria aguda e, inclusive, en los lugares donde es prevalente, con parasitosis intestinal. Otros cuadros a diferenciar son cólico renal o vesicular, además de envenenamiento por otros tóxicos $\left({ }^{18}\right)$.

En el campo neurológico, la parálisis causada por la neuritis saturnina debe ser diferenciada de otras neuritis, como la arsenical, la infecciosa del síndrome de Guillain-Barré, la de diabetes y, en nuestros países, la neuritis por desnutrición. Éstas difieren de la parálisis por plomo en que tienen localizaciones atípicas y componente sensorial, mientras que la del plomo involucra los nervios motores de los grupos musculares más usados. Un caso especial lo constituye la que se ha dado en llamar "parálisis del sábado por la noche", que es indolora, bilateral, compromete sólo a los radiales y generalmente hay antecedente de alcoholismo. La encefalitis por plomo en el trabajador expuesto es muy rara en las actuales condiciones de trabajo. Sin embargo, de sospecharse, se la debe diferenciar de hipertensión endocraneana, meningitis séptica y aséptica, neoplasias y neuroparasitosis. Al margen, y por su relativa frecuencia entre nosotros, no podemos dejar de mencionar en el diagnóstico diferencial casos de "ganancia secundaria", por su implicancia médico legal y no por su importancia toxicológica $\left({ }^{18}\right)$.

El diagnóstico diferencial se debe basar en: 1) Antecedente laboral; 2) Historia clínica y de antecedentes, acuciosas; y, 3) Exámenes de laboratorio positivos. No obstante, el mayor problema se presenta cuando enfrentamos manifestaciones agudas que 
requieren de decisiones rápidas, con tiempo muy limitado para realizar exámenes de laboratorio, que casi siempre no están a la mano en la empresa ni en el hospital de referencia local. Este problema es responsable a menudo de las ya mencionadas laparotomías iatrogénicas, amén del retraso en el tratamiento $\left({ }^{1}\right)$.

Una situación no poco frecuente ocurre cuando un trabajador expuesto a plomo desarrolla sintomatología que, si bien es cierto podría deberse a plomo, también puede ser causada por otra enfermedad, mas aún si hallamos que el trabajador presenta absorción aumentada de plomo y alguna alteración de laboratorio. En estos casos, siempre recordar que laborar expuesto a plomo no protege al trabajador de contraer otras enfermedades. Por consiguiente, siempre es necesario considerar exámenes para confirmar o excluir una enfermedad diferente al saturnismo, ejemplo, un simple déficit en la ingesta de hierro puede causar también alteraciones hemáticas. Otras veces, al creernos "fracasados" en diagnosticar el cuadro clínico bizarro de un trabajador expuesto a plomo, podríamos terminar diagnosticando "intoxicación por plomo". En este caso, tengamos presente que un trabajador expuesto tiene el $\mathrm{Pb}-\mathrm{S}$ cercano o ligeramente mayor a $40 \mathrm{ug} / 100$, entonces, el médico debe recordar que la intoxicación por plomo siempre es un cuadro bien definido y no una serie de manifestaciones inconexas o vagas $\left({ }^{1,18,26}\right)$.

Es frecuente el dilema médico legal para dilucidar si una cantidad equis de $\mathrm{Pb}-\mathrm{S}$ documentada en un trabajador por exposiciones pasadas es la causa de alguna intoxicación antigua que reclama. Si ya pasaron varios años desde la exposición, el caso es muy difícil de resolver, pues toda prueba específica actual es probable que sea normal, incluyendo el $\mathrm{Pb}-\mathrm{S}$. Ante este dilema, al médico sólo le queda averiguar la carga corporal de plomo, es decir, la cantidad de plomo que el organismo -principalmente el tejido óseo- pueda aún atesorar, teniendo en cuenta que la vida media del plomo varía entre 15 y 27 años. La prueba a aplicar se llama "eliminación provocada de plomo" y consiste en administrar edetato disódico monocálcico para liberar el plomo por la orina y poner en evidencia exposición alta en el pasado. Esta prueba suele complementarse con la medición de ZPP/FEP, pues la FEP permanece alta durante mucho tiempo después de cesar la exposición. Con todo, una prueba positiva sólo nos dice de exposiciones pasadas, pero no puede darnos luz sobre intoxicaciones antiguas del trabajador $(1,18,33)$.

\section{TRATAMIENTO DE LA INTOXICACIÓN POR PLOMO}

Quelación es la acción médica por la cual se usa ciertas drogas llamadas agentes quelantes, por vía oral o endovenosa, para reducir la cantidad de plomo absorbidas por los tejidos del organismo. Los agentes quelantes de mayor uso continúan siendo el edetato disódico monocálcico ( $\left.\mathrm{CaNa}_{2} \mathrm{EDTA}\right)$, cuyo nombre comercial es calcio disodio versenate ${ }^{R}$ o simplemente Versenate $^{\mathrm{R}}$, y la D-penicilamina de nombre comercial Pencillamin $^{\mathrm{R}}$ o Cuprimine ${ }^{\mathrm{R}}$. Desde 1990, han aparecido nuevas drogas para el tratamiento de la intoxicación, tal como el Succimer ${ }^{\mathrm{R}}$, ácido 2, 3 dimercaptosuccinico o DMSA y su sucedáneo, el ácido 2, 3 dimercapto-1propano-sulfónico, DMPS, que han mejorado las opciones terapéuticas, son menos tóxicos, más fáciles de administrar y sobre todo no causan depleción de cationes $\left({ }^{38-41}\right)$.

El edetato, clásico agente quelador del plomo, se presenta en tabletas de 0,5 g y ampollas de $5 \mathrm{~mL}$ en solución al $20 \%$ y aunque no está bien dilucidado su mecanismo para eliminar el plomo de los tejidos, se le administra por vía oral a razón de $20 \mathrm{mg} / \mathrm{kg} \mathrm{q} 8 \mathrm{~h}$ por 5 días. Si es necesario otro curso, debe intercalarse 3 días. Vía endovenosa se administra de 15 a $25 \mathrm{mg} /$ $\mathrm{kg}$ en solución de dextrosa al $5 \%$ para pasar en una o dos horas dos veces al día; en este caso, la dosis total no debe exceder $50 \mathrm{mg} / \mathrm{kg} /$ día y se da en cursos de 5 días con un lapso de descanso de por lo menos 2 días entre cada curso. Analizar la orina a diario y discontinuar el tratamiento si aparece alguna alteración. No se debe dar este antídoto en caso de hipertensión endocraneal $\left({ }^{1,40}\right)$.

La penicilamina actúa aumentando la excreción urinaria de plomo, pero no tan específicamente como el edetato. No se conoce ni su mecanismo ni su lugar específico de acción. Se presenta en cápsulas de 125 y $250 \mathrm{mg}$, se la administra vía oral de 25 a $35 \mathrm{mg} / \mathrm{kg} /$ día, en dosis divididas, iniciando con la dosis más baja e incrementándola progresivamente $\left(^{1,41}\right)$.

El Succimer ${ }^{R}$ es un análogo de la British anti lewisite, BAL, y está disponible en cápsulas de 100 $\mathrm{mg}$, para administrar inicialmente $10 \mathrm{mg} / \mathrm{kg}$ cada 8 horas por 5 días, continuar luego con $10 \mathrm{mg} / \mathrm{kg}$ cada 12 horas por 14 días más, 19 días en total. Si se necesita un nuevo curso, intercalar 2 semanas. El DMPS ha 
sido administrado con éxito en intoxicación industrial por plomo y aunque algunas veces se ha descrito desarrollo del síndrome de Stevens Jonhson, parece ser que en el futuro será el medicamento de elección en esta patología. El uso de quelantes ha sido revisado por la Agencia para Seguridad y Salud Ocupacional (OSHA) y la Conferencia Americana de Higienistas Industriales Generales (ACGIH) y su aplicación continúa vigente con algunas limitaciones $(1,39,40,42,44)$.

La experiencia acumulada por la investigación médica en el uso de quelantes ha encontrado un punto de equilibrio entre ventajas y desventajas, estableciendo cuándo y en qué circunstancias es aceptable: ha confirmado su efectividad en el tratamiento de la intoxicación aguda y severa, pero definitivamente ha proscrito su uso como profiláctico y para el tratamiento de exposición a plomo a largo plazo, debido a sus numerosos efectos adversos y dañinos asociados a ellos. Así, la norma OSHA excluye su uso como profilácticos, poniendo fin a una larga historia de uso y abuso de esta terapia en algunas empresas mineras y metalúrgicas, incluyendo su uso rutinario para disminuir los niveles de $\mathrm{Pb}-\mathrm{S}$ en trabajadores expuestos.

Por otro lado, el aplicar terapia de quelación como única medida, es inaceptable. Y es que el tratamiento médico ocupacional debe pasar por indicar mejoría en las condiciones ambientales del área de trabajo y luego, si todavía es necesario, recién "quelar" al trabajador; salvo en la intoxicación grave, en que se debe proceder de inmediato. Luego que se logre disminuir los niveles de $\mathrm{Pb}-\mathrm{S}$ y otros indicadores de toxicidad, el trabajador solamente debe retornar al trabajo si el $\mathrm{Pb}-\mathrm{A}$ en su área de labor se encuentra bajo el valor límite umbral (VLU o TLV, por sus siglas inglesas) y, por tanto, ya no representa riesgo de reintoxicación. Caso contrario, el trabajador debe ser reubicado en una zona donde el plomo y otros xenobióticos neuro o nefrotóxicos ambientales se encuentren bajo el VLU. Por último, la norma recomienda aplicar la terapia quelante en trabajadores expuestos cuando el $\mathrm{Pb}-\mathrm{S}$ excede $80 \mathrm{ug} /$ 100 y se acompaña de sintomatología franca. Sin embargo, con la mejora tecnológica de los procesos metalúrgicos y las medidas de higiene colectivas e individuales aplicadas a las áreas de labor, ya rara vez encontramos $\mathrm{Pb}-\mathrm{S}$ mayor de $50 \mathrm{ug} / 100\left({ }^{1,18,42,44,45}\right)$. Tabla 3.

Respecto a la llamada prueba diagnóstica, y sólo para fines médico legales, la norma permite aplicar
Tabla 3. Norma OSHA para manejo de plomo sanguíneo en expuestos.

Nivel de $\mathrm{Pb}-\mathrm{S}$ requerido para cambio de lugar de labor (debe confirmarse con una segunda muestra dentro de las 2 semanas del primer informe):

$>60$ ug/100 g de promedio en las 3 últimas muestras ó 50 ug/100 g o más de todas las muestras en los últimos 6 meses, aunque la última muestra sea 40 ug/100 g o menos

Frecuencia con que los trabajadores expuestos al nivel de acción (30 ug/m $\mathrm{m}^{3}$ TWA) deben chequearse el $\mathrm{Pb}-\mathrm{S}$ (Es recomendable dosar $\mathrm{ZPP}$ cada vez que se valore $\mathrm{Pb}-\mathrm{S}$ ):

1. Nivel del último Pb-S: < 40 ug/100: Cada 6 meses

2. Nivel entre 40 y 60 ug/100: Cada 2 meses

3. Trabajador retirado del área de exposición por niveles elevados de $\mathrm{Pb}-\mathrm{S}$ : Cada mes

Nivel de acción de $\mathrm{Pb}-\mathrm{A}$ para que el trabajador sea retirado del área de exposición por niveles elevados de $\mathrm{Pb}-\mathrm{S}$ (sin considerar protección individual con respirador): $30 \mathrm{ug} / \mathrm{m}^{3} 8 \mathrm{~h}$ TWA

Vuelta al trabajo: Nivel de Pb-S (confirmado con $2^{\mathrm{a}}$ muestra) con el cual el trabajador puede volver al trabajo, si las condiciones ambientales han mejorado: $<40 \mathrm{ug} / 100 \mathrm{~g}$

Adaptado de: US Department of Labor, Occupational Safety and Health Administration: Lead Standard, 20 CFR 1910.1025 (Revised) Washington, DC: US Government Printing Office. 1990.

quelantes si se les administra bajo vigilancia o supervisión de un médico toxicólogo u ocupacionista. La norma recomienda que cuando se decida aplicar quelación, se debe notificar por escrito al trabajador indicándole el potencial efecto dañino del quelante y su derecho a obtener una segunda opinión $\left({ }^{44}\right)$.

\section{MONITOREO BIOLÓGICO DE LA EXPOSICIÓN A PLOMO}

En el Perú, desde antes de los años cincuenta del siglo pasado, la preocupación por monitorear la salud del trabajador expuesto a plomo con indicadores biológicos de exposición ha sido constante. Entonces el plomo en sangre ya era útil, aún cuando su método analítico (Ditizona) no era suficientemente fidedigno. Por entonces, el diagnóstico se complementaba con indicadores que hoy están en desuso, como reticulocitosis, volumen globular de hematíes, 
hemoglobina corpuscular media, punteado basófilo de hematíes y otros; de ellos aún usamos con éxito la hemoglobina $(\mathrm{Hb})$. Hoy existen otros IBE de uso más o menos común en la monitorización del trabajador expuesto. Uno de los más importantes continúa siendo el $\mathrm{Pb}-\mathrm{S}$ analizado ahora por absorción atómica, pues nos da una visión bastante directa de exposición reciente y de carga corporal. El antiguo BEI hemoglobina y el nuevo ZPP nos informan del daño bioquímico producido por la exposición. Consideraciones económicas y la disponibilidad de laboratorios calificados algunas veces entorpecen la indicación de estos BEIs; sin embargo esas preocupaciones nunca deben ser antepuestas a la salud del trabajador $\left({ }^{46,47}\right)$.

Los Criterios OSHA para exposición a plomo requieren que $\mathrm{Pb}-\mathrm{S}$ y $\mathrm{ZPP}$ "se realicen regularmente en todo trabajador expuesto. En aquellos que estén expuestos a plomo ambiente mayor a $30 \mathrm{ug} / \mathrm{m}^{3}$ por más de 30 días por año debe dosarse $\mathrm{Pb}-\mathrm{S}$ y ZPP por lo menos cada 6 meses. $\mathrm{Si}$ el $\mathrm{Pb}-\mathrm{S}$ hallado es mayor o igual a $40 \mathrm{ug} / 100 \mathrm{~g}$ de sangre entera, equivalente a 42 $\mathrm{ug} / 100 \mathrm{~mL}$, se le debe examinar cada 2 meses hasta que el plomo baje a niveles menores de $40 \mathrm{ug} / 100 \mathrm{~g}$ " (Tabla 3). El criterio OSHA para la separación del lugar de trabajo es de $50 \mathrm{ug} / 100 \mathrm{~g}$ ó $53 \mathrm{ug} / 100 \mathrm{~mL}$. La norma también indica criterios de acción rápida, máximo dos semanas para cuando el $\mathrm{Pb}-\mathrm{S}$ exceda el criterio de separación del lugar de trabajo y recomienda a las empresas incluir en sus programas de control a todos los trabajadores expuestos, tanto aquellos que laboren directamente en plomo como a los que tienen un contacto incidental con el metal $(25,44,46,47)$.

Resumiendo, un trabajador puede continuar laborando con valores de hasta $50 \mathrm{ug} / 100 \mathrm{~g}$. Cuando alcanza o excede este valor, la acción a tomar es retirarlo de la exposición y transferirlo a un área limpia. Para que vuelva a su labor habitual se debe esperar que el nivel del $\mathrm{Pb}-\mathrm{S}$ retorne a $40 \mathrm{ug} / 100 \mathrm{~g}$. Pero, en la práctica esto puede requerir meses o incluso años, pues el plomo de la carga corporal atesorado en los huesos toma mucho tiempo para liberarse e impide que el $\mathrm{Pb}-\mathrm{S}$ rápidamente llegue a un nivel aceptable. Además, se ha observado que los trabajadores con carga corporal alta son más sensibles a elevaciones intercurrentes de $\mathrm{Pb}-\mathrm{S}$, por lo que algunas veces puede ser necesario retirarlos definitivamente de la exposición $\left({ }^{42,44}\right)$ (Tabla 3).
La ventaja de combinar los indicadores $\mathrm{Pb}-\mathrm{S}$, ZPP y hemoglobina, es que los tres pueden ser obtenidos de una sola muestra de sangre entera. La inclusión de ZPP en la batería tiene además la ventaja de identificar a trabajadores que reaccionan más sensiblemente al plomo y no nos limita al margen de seguridad de $\mathrm{Pb}$ S. La norma OSHA deja casi fuera la determinación de AAL urinario y la dehidratasa del AAL en sangre. Sin embargo, estas pruebas son sensibles a cambios sutiles en la biosíntesis del Hem y ayudan a discriminar falsos positivos. El plomo ambiental, humos o fumes, también es un buen indicador de riesgo, incluyendo al polvo de plomo que podría ser ingerido al hablar o al comer con las manos contaminadas, contribuyendo así a la carga corporal de plomo. En la Comunidad Europea, la norma requiere supervisión biológica, incluyendo determinación del $\mathrm{Pb}-\mathrm{S}$ una vez cada 6 meses y similar criterio que OSHA para separación del lugar de trabajo $\left({ }^{32,39,44-47}\right)$.

El plomo urinario $\mathrm{Pb}-\mathrm{U}$, que aún se usa como IBE en algunos centros minero-metalúrgicos, es una prueba con mucha más variabilidad que el $\mathrm{Pb}-\mathrm{S}$ y sólo se la indica usar cuando se emplee quelantes para verificar la eliminación del plomo por el medicamento. La medición de plomo en pelo y uñas actualmente no tiene ninguna aplicación práctica en la valoración de la exposición ocupacional. No usar nunca agentes quelantes como profilácticos en exposición a plomo, porque podríamos desencadenar una enfermedad renal iatrogénica. Es más, los quelantes también "quelan" oligoelementos esenciales, produciendo efectos que en sí mismo representan un riesgo $\left({ }^{18,46,47}\right)$.

Indicador útil en la valoración de la toxicidad renal causada por el xenobiótico es la enzima N-acetil-betaglucosamidasa (NAG), que parece ser el más sensitivo de los indicadores cuando el Pb-S alcanza los $80 \mathrm{ug} /$ 100 g. Con éxito, se usa en exposición industrial a plomo, aunque a niveles bajos la relación $\mathrm{Pb}-\mathrm{S}$ y NAG sea poco consistente $\left({ }^{18}\right)$.

En cuanto a lesión renal, el reciente uso específico de las glutation S-transferasas (GS-T) $\alpha$ GS-T y $\beta$ GS-T en orina ha venido a resolver muchas controversias, pues son indicadores tempranos del lugar y magnitud del daño renal. La $\alpha \mathrm{GST}$ es muy específica para determinar lesión del túbulo proximal, mientras que la $\beta$ GST lo es para el túbulo distal $\left({ }^{48}\right)$. 
Tabla 4. Valores umbrales límites de plomo en ambiente e índices biológicos de exposición.

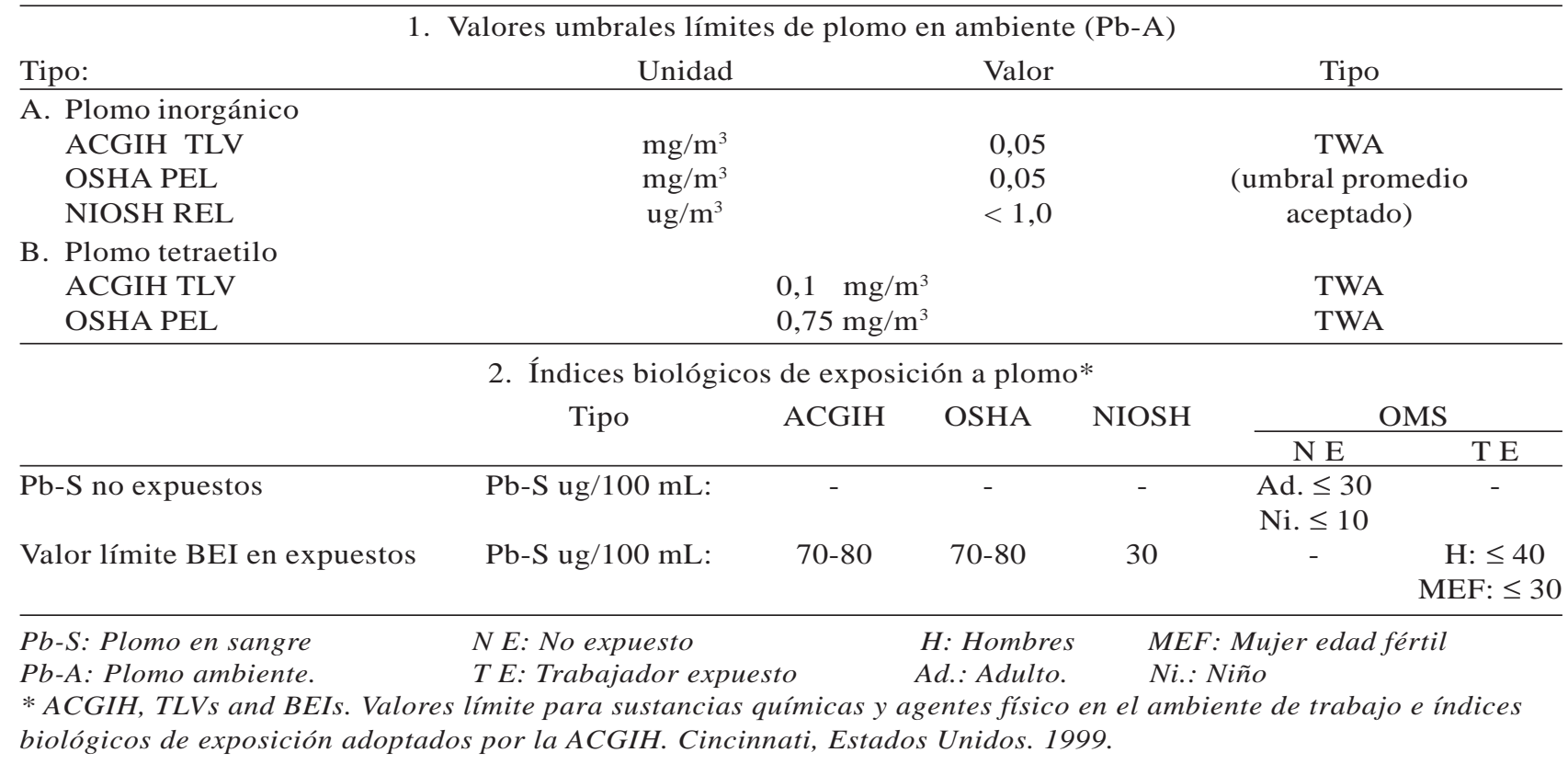

Para valorar daño en el sistema nervioso periférico, la recomendación es medir la velocidad de conducción del impulso nervioso, pues con $\mathrm{Pb}-\mathrm{S}$ entre 40 y $60 \mathrm{ug} /$ $100 \mathrm{~mL}$, se ha detectado disminución de la velocidad del impulso nervioso. Finalmente, no podemos dejar de referir el uso la densitometría ósea y su examen por fluorescencia a los rayos equis, como indicador de acumulación de plomo en huesos, pero sin aplicación práctica en el manejo de la enfermedad ocupacional $\left({ }^{46,47}\right)$.

Para valores referenciales de ZPP y hemoglobina, OSHA no ha establecido límite, por lo que el médico ocupacionista debe referirlas a su juicio clínico y experiencia. Es importante recordar que nuestras más grandes industrias de plomo se encuentran en minería y que la minería en el Perú está, frecuentemente, por encima de $3000 \mathrm{msnm}$. Por tanto, el valor de hemoglobina normal es, para varones, de 15 a $18 \mathrm{~g} \%$. En la altura, en expuestos a plomo que tengan hemoglobina reiteradamente menor a $13 \mathrm{~g} / 100$, es necesario investigar otras causas de anemia y descartarlas y luego considerar esta intoxicación, recordando que el valor de la hemoglobina también varía por causas ajenas al plomo, como estado nutricional o parasitosis, por ejemplo. En cuanto a la zinc proto porfirina, se debe considerar un valor de $500 \mathrm{ug} / 100 \mathrm{~mL}$ como el valor permisible más alto para un trabajador con $\mathrm{Pb}-\mathrm{S} 50 \mathrm{ug} / 100 \mathrm{~mL}\left({ }^{1,7,26}\right)$.

Por excepción, en el monitoreo biológico de plomo alquilo usa como IBE el plomo urinario $(\mathrm{Pb}-\mathrm{U})$, cuyo nivel debe mantenerse por debajo de $150 \mathrm{ug} / \mathrm{L}$. En el caso de los plomos alquilos, no existen datos fehacientes para el manejo útil del indicador plomo sanguíneo $\left({ }^{1,43,47}\right)$ (Tablas 2 y 4$)$.

Unas palabras para concluir el aspecto del monitoreo biológico. La norma OSHA para retiro temporal o permanente de un trabajador se reduce sólo a un esquema frío sin considerar el aspecto humano que hay detrás. La empresa, por otro lado, tampoco debe considerar al retiro de un trabajador por $\mathrm{Pb}-\mathrm{S}$ elevado como una "catástrofe" en sí, sino más bien, como una oportunidad adicional para mejorar las condiciones higiénicas del ambiente de trabajo.

Finalmente, enfatizamos que no existe dieta, alimento (leche), vitamina u otra preparación medicinal para "prevenir" la intoxicación ocupacional por plomo. El único medio aceptado es el control higiénico y técnico del ambiente de trabajo y del trabajador $\left(^{1,18}\right)$. 


\section{EXPOSICIÓN OCUPACIONAL A COMPUESTOS ALQUILOS DE PLOMO}

En Salud Ocupacional, los únicos compuestos alquilos de plomo de importancia clínica son el plomo tetraetilo (PTE) y el plomo tetrametilo (PTM), usados como agentes antidetonantes de combustibles fósiles. El PTE se introdujo hacia 1923 y el PTM en 1959. Son plomos orgánicos que tienen la propiedad común de ser líquidos liposolubles. El punto de ebullición (PE) del PTM es $110^{\circ} \mathrm{C}$ y es más volátil que el PTE cuyo $\mathrm{PE}$ es $199^{\circ} \mathrm{C}$. Ambos se usa exclusivamente como antidetonantes, pero su volumen de uso mundial es enorme, aproximadamente 46000 toneladas métricas únicamente en USA (1985), cantidad sólo superada por la industria de fabricación de baterías $\left({ }^{1,43}\right)$.

\section{EXPOSICIÓN}

La exposición está ligada a su fabricación, distribución y manejo en las plantas de producciónrefinado y a su transporte a las refinerías de combustibles. En estos lugares, el riesgo es bajo por las estrictas medidas de control usadas. Sin embargo, la exposición de importancia toxicológica ocupacional la encontramos en: 1) Limpieza y reparación de tanques contenedores de gasolina con plomo en las estaciones de distribución y venta al por menor de combustibles, denominados entre nosotros "grifos", sobre todo porque los tanques de almacenamiento a menudo contienen en el fondo lodos sedimentados con un alto contenido de plomo alquilo; 2) Al momento del despacho de combustibles, pues la gasolina es muy volátil; aquí, la vía de ingreso es doble, cutánea y respiratoria y es de nuestro diario conocer que son muy escasos los trabajadores de "grifo" que usan equipo de protección. Por la misma razón, otro punto ocupacional de posible intoxicación está en los talleres de mecánica sin ventilación adecuada que usan gasolina con plomo para desengrasar o limpiar piezas; 3) Finalmente, y aunque no ocupacional, no olvidar al grupo tóxico-adicto de "oledores de gasolina" $\left({ }^{43}\right)$.

\section{TOXICOLOGÍA}

El efecto toxicológico de los compuestos orgánicos del plomo se debe a su alta solubilidad en las grasas que los hace fácilmente absorbibles a través de la piel intacta y mucosas, además de facilitarles el paso a través de la barrera hemato-encefálica. Gran parte de los compuestos alquilos de plomo se fija en la fracción grasa del organismo; los PTE y los PTM tienen 3 veces más afinidad por las grasas que el plomo inorgánico. Luego, y por la misma razón, una vez absorbidos se dirigen a órganos con alto contenido graso, como los del sistema nervioso central. Su metabolismo se realiza en el hígado que los transforma en plomo inorgánico y plomo trialquilo, compuesto hidrosoluble. Investigaciones recientes indican que realmente el PTM es el responsable de las manifestaciones tóxicas del envenenamiento por PTE. En ratas se ha demostrado que la dosis letal de PTE es aproximadamente $11 \mathrm{mg} /$ $\mathrm{kg}$ y la del PTM, $83 \mathrm{mg} / \mathrm{kg}$; por tanto existe una diferencia de 7 a 1 en la toxicidad de estos compuestos. Esta diferencia "compensaría" el riesgo producido por la mayor volatilidad del PTM.

El estándar OSHA para PTE y PTM es de $75 \mathrm{mg} /$ $\mathrm{m}^{3}$ TWA y PEL (umbral promedio aceptado referido a nivel de exposición permisible). Con relación a ocupación, la intoxicación por plomo orgánico requiere exposición suficiente, es decir, al considerar el diagnóstico se debe contar con antecedente de exposición prolongada y específica o, en todo caso, permanencia laboral constante en una atmósfera que contenga concentraciones altas de los compuestos. Si el tiempo de exposición es sólo de algunas horas, para que haya intoxicación el ambiente tendría que haber superado los $10 \mathrm{mg} / \mathrm{m}^{3}$. Sin embargo, siempre el mayor tiempo de exposición a concentraciones bajas es más peligroso que un tiempo corto en alta exposición $\left({ }^{43}\right)$ (Tabla 2 y 4 ).

\section{CUADRO CLÍNICO}

La intoxicación por plomo orgánico se inicia con síntomas no específicos que en casos leves pueden autolimitarse y "cesar" en algunos días. Sin embargo, estos síntomas, que podríamos llamar prodrómicos o «centinelas», son sólo el inicio de un proceso mucho más severo. Después de exposiciones levesprolongadas o severas-cortas, la intoxicación por PTE suele tener un inicio súbito. Como regla general, el lapso entre exposición y ataque con síntomas "centinela" está en razón inversa a la severidad de la enfermedad, es decir, a menor tiempo de inicio, intoxicación más severa. La intoxicación de mediana 
intensidad, se caracteriza por irritabilidad, inestabilidad emocional, insomnio y otros problemas del sueño, actividad física aumentada pero errática, síntomas gastrointestinales que incluyen anorexia, náuseas, vómitos y ocasionalmente diarrea.

Al examen, el trabajador presenta palidez, baja de presión arterial, temblor marcado y reflejos osteotendinosos aumentados. En los casos más severos, la fase prodrómica se presenta a veces con síntomas más o menos violentos que se instalan en forma progresiva; pero, la mayoría de veces el inicio es abrupto con gritos y risas inmotivados, hiperactividad física sin objeto, alucinaciones y deluciones. Sin un buen diagnóstico, estos síntomas pueden persistir varios días e inclusive llevar a consulta siquiátrica cuando no al coma y muerte. La diferencia más importante entre la intoxicación por plomo alquilo e inorgánico es la ausencia de alteraciones hemáticas y en la síntesis de núcleo hem en el caso del $\mathrm{Pb}$ alquilo y ésta es precisamente la razón por la que estos indicadores no tienen valor diagnóstico o preventivo en la intoxicación por PTE. El diagnóstico diferencial sigue las mismas pautas que las del plomo inorgánico, con énfasis en el aspecto neurológico y en cuadros psiquiátricos primarios $\left({ }^{1,18,43}\right)$.

\section{TRATAMIENTO}

No se conoce terapia específica, por lo que además del cese de la exposición el tratamiento es sintomático. El cuadro de agitación puede ser controlado con sedación a base de diazepam y mantenimiento del equilibrio hidroelectrolítico. Aún con el cese de la exposición y un buen control del paciente, la recuperación puede tardar semanas o meses. El uso de quelantes, pueda que produzca un ligero aumento en la excreción del xenobiótico, pero no tiene efecto cierto en la recuperación del paciente $\left({ }^{1,18,43}\right)$.

\section{PREVENCIÓN}

Al no existir un medicamento específico para tratar la intoxicación por plomo alquilo, la acción del médico ocupacionista se enfocará al aspecto preventivo. No todas las empresas, los "grifos" principalmente, pueden disponer de medios técnicos adecuados para implementar normas de higiene, pero sí pueden y deben proporcionar al trabajador protección personal, como ropa adecuada para el trabajo que realizan e implementar acciones preventivo promocionales de salud e higiene en el trabajo, además del examen ocupacional periódico que incluya monitoreo biológico. Se debe controlar exhaustivamente la limpieza de tanques que han contenido gasolina con plomo, pues, amén de ser un trabajo en espacio confinado, existe riesgo de intoxicación aguda con plomo alquilo. Por ello se le considera un trabajo de alto riesgo y, de no contarse con un higienista que supervise esta acción, debería prohibirse el ingreso al tanque sin ropa de protección de cuerpo entero y sin una línea autosuficiente de aire $\left(^{1,43,46}\right)$.

\section{REFERENCIAS BIBLIOGRÁFICAS}

1. Zenz C, Ed. Occupational medicine: principles and practical applications. $2^{\text {nd }}$ Ed. Chicago: Mosby; 1988.

2. Hunter D. The Disease of Occupations. $4^{\text {th }}$ Ed. Aulesbury: English Universities Press by Hazell Watson \& Viney; 1969.

3. Bellinger DC, Stiles KM, Needleman HL. Low-level lead exposure, intelligence and academic achievement: A longterm follow-up study. Pediatrics. 1992;90:855.

4. Daines RH, Smith DW, Feliciano A, Trout JR. Air levels of lead inside and outside of homes. Ind Med Surg. 1972;41:26.

5. Centers for Disease Control and Prevention (CDC). Preventing lead poisoning in young children: A statement by the Centers for Disease Control. Atlanta, Georgia: US Department of Health and Human Services, Public Health Service, 1991.

6. Jawrowski Z. Stable lead in fossil ice and bones. Nature. 1968;217:152.

7. Ramírez AV, Cam PJ, Medina M. Plomo sanguíneo en los habitantes de cuatro localidades peruanas. Rev Panam Salud Pública. 1997 Mayo;1(5): 344-8.

8. Parmeggiani L, Ed. Encyclopaedia of Occupational Health and Safety. $3^{\text {th }}$ Ed. Geneva: International Labour Office; 1989.

9. Jelinek CF. Levels of lead in the United States food supply. J Assoc Anal Chem. 1982;65:942.

10. Moore JE. Community aspects of childhood lead poisoning. Am J Public Health. 1970;60:1430.

11. Dix T, Walker S, Cosby D, Woernle CH. Elevated blood lead levels associated with illicitly distilled alcohol Alabama, 1990-1991. MMWR. 1992;41:294-5.

12. Wedeen RP. Poison in the pot: the legacy of lead, Carbondale -Edwardsville, ll. Southern Illinois: University Press; 1984.

13. Piomelli S, Wolff JA. Childhood lead poisoning in the ' 90 s. Pediatrics. 1994;93:508-10.

14. Amitai Y, Graef JW, Brown MJ, Gerstle RS, Kahn N, Cochrane PE. Hazards of 'deleading' homes of children 
with lead poisoning.. Am J Dis Child. 1987 Jul;141(7):75860.

15. Norton RL, Kauffman KW, Chandler DB, Burton BT, Gordon J, Fostern LR. Intravenous lead poisoning associated with methamphetamine use. Vet Hum Toxicol. 1989;31:379.

16. Kikano GE, Stange KC. Lead poisoning in a child alter a gunshot injury. J Fam Pract. 1992;34:498-504.

17. US Environmental Protection Agency. Maximum contaminant level goals and national primary drinking water regulations for lead and copper. Federal Register. 1991;56:26469-26470.

18. Ellemhorn MJ. Medical Toxicology. $2^{\text {nd }}$ Ed. Baltimore: Williams Wilkins; 1996.

19. Levy BS, Wegman DH, Eds. Occupational Health. Boston: Little, Brown and Co.; 1988.

20. Friberg L, Nordberg GF, Vouk VB, Eds. Handbook of toxicology of metals. Amsterdam: Elsevier; 1979.

21. Hemberg S. Lead. En: Zenz C, Ed. Occupational medicine: principles and practical applications. $2^{\text {nd }}$ Ed. Chicago: Mosby; 1988.

22. Kehoe RA. Occupational lead poisoning. 2. Chemical signs of the absorption of lead. J Occup Med. 1972;14:390.

23. Ramírez AV, Tapia G. Cuadro clínico del Saturnismo en la industria metalúrgica de altura. Rev Bras Saúde Ocup. 1998;24:85-94.

24. Lerner S, Gartside P, Roy B. Free erythrocyte protoporphyrin, zinc protoporphyrin and blood lead in newly re-exposed smelter workers: a prospective study. Am Ind Hyg Assoc J. 1982;43:516-9.

25. Lauwerys R, Rand R, Hoet P, editors. Industrial chemical exposure: guidelines for biological monitoring. Boca Raton: Lewis Publishers; 1993.

26. Saryan LA. Blood lead monitoring for worker protection. Battery Man. 1988;30(6):25.

27. Piomelli S. Free erythrocyte porphyrins in the detection of undue absorption of $\mathrm{Pb}$ and of Fe deficiency. Clin Chem. 1977;2:264-9.

28. Schirmer J, Anderson HA, Saryan LA. Fatal pediatric poisoning from leaded paint -Wisconsin, 1990. MMWR. 1991;40:193-5.

29. Graef J. Lead poisoning. Part I. Clinical Toxicology Review. 1992;14(8):1-2.

30. Lane RE. The care of the lead worker. Br J Ind Med. 1993;50:193.

31. World Health Organization. Environmental health criteria 3, lead. Geneva: World Health Organization; 1977.

32. Saryan LA. Protoporphyrin (FEP/ZPP) screening in industrial lead exposure. Battery Man. 1988;30(1):16.

33. Saryan LA. Testing for protporphyrin (ZPP/FEP) in the brass and bronze foundry. The Crucible; 1989. p. 6.
34. Lamola AA, Yamane T. Zinc protoporphyrin, in the patients with lead intoxication and iron deficiency anaemia. Science. 1974; $186: 936$.

35. Roels HA, Lauwerys RR, Buchet JP, Vrelust MT. Response of free erythrocyte porphyrin and urinary d-aminolevulinic acid in men and women moderately exposed to lead. Int Arch Arbeitsmed. 1975;34:97.

36. Boothby JA, de Jesus PV, Rowland LP. Reversible factor of motor neuron disease. Arch Neurol. 1974;21:18-23.

37. Cantarow A, Trumper M. Lead poisoning. Baltimore: Williams \& Wilkins; 1944.

38. Fine JS, Goldfrank LR. Update in medical toxicology. Pediatric Clin North Am. 1992;39:1031.

39. Lundberg P, Ed. Scientific basis for Swedish Occupational Standards XII (Consensus report for inorganic lead, September 5. 1990). Arbete Och Halsa. 1992;6:13.

40. Chisolm JJ Jr. BAL, EDTA, DMSA and DMPS in the treatment of lead poisoning in children. Clin Toxicol. 1992;30:493-504.

41. Shannon M, Graef J, Lovejoy FH. Efficacy and toxicity of d-penicillamine in low-level lead poisoning. J Pediatr. 1988; $12: 799-804$.

42. ACGIH, TLVs and BEIs: Threshold Limits Values for Chemical Substances and Physical Agents. Biological Exposure Indices. Cincinnati: ACGIH; 2003.

43. Cremer JE. Toxicology and biochemistry of alkyl lead compounds. Occup Health Rev. 1965;17:14.

44. US Department of Labor, Occupational Safety and Health Administration. Lead Standard, 20 CFR 1910.1025. Washington, DC: US Government Printing Office; 1990.

45. Graef J. Lead poisoning management II. Clin Toxicol Rev. 1992;14(9):1-2.

46. Levy VS, Wegman DH, Ed. Occupational Health. Recognizing and Preventing Work-Related Disease. Boston,: Little, Brown and Co.; 1988.

47. La Dou J. Medicina Laboral y Ambiental. 2 a Ed. México DF: Ed. Manual Moderno; 1999.

48. Harrison DJ, Kharbanda R, Scout-Cummingham D, McClellan LI, Hayes JD. Distribution of glutathione Stransferases isoenzime in human kidney: basis for possible markers of renal injury. J Clin Pathol. 1989;42:624-9.

Manuscrito recibido el 17 de febrero de 2005 y aceptado para publicación el 15 marzo 2005.

Correspondencia: Dr. Augusto V. Ramírez

Las Mandarinas 210-Ap. 306

Correo-e: augustovram@yahoo.es 